\title{
Editorial
}

\section{Dietary Fiber: Chemistry, Structure, and Properties}

\author{
Qingbin Guo, ${ }^{1,2}$ Ji Kang $\mathbb{D}^{1,2}$ Yanjie Bai $\mathbb{D}^{3}{ }^{3}$ and Feng $X u^{4}$ \\ ${ }^{1}$ State Key Laboratory of Food Nutrition and Safety, Tianjin University of Science \& Technology, Tianjin 300222, China \\ ${ }^{2}$ Department of Food Science, University of Guelph, Ontario, Canada N1G 2W1 \\ ${ }^{3}$ School of Public Health, Soochow University, Suzhou, China \\ ${ }^{4}$ Merck \& Co., Kenilworth, NJ 07033, USA \\ Correspondence should be addressed to Ji Kang; jkang12@uoguelph.ca
}

Received 18 July 2018; Accepted 18 July 2018; Published 26 September 2018

Copyright (c) 2018 Qingbin Guo et al. This is an open access article distributed under the Creative Commons Attribution License, which permits unrestricted use, distribution, and reproduction in any medium, provided the original work is properly cited.

Dietary fiber is defined as "the edible parts of plants or analogous carbohydrates that are resistant to digestion and absorption in the human small intestine with complete or partial fermentation in the large intestine." It includes mainly non-starch polysaccharides, oligosaccharides, resistant starch, and lignins. Health benefits of dietary fiber including promoting laxation, attenuating blood cholesterol and glucose, and preventing certain cancers have been extensively studied by many human and animal studies. These health benefits may be attributed to several physiological properties such as bulking, water-holding capacity, cation- and cholesterol-binding properties, and fermentation properties. However, the detailed mechanisms of its health benefits are still not clear which deserve more investigations. In addition, the food application of dietary fiber is still facing many challenges including poor texture and unpleasant mouthfeel of the fiber-fortified food products. Journal of Chemistry set out to publish a special issue devoted to the topic "Dietary Fiber: Chemistry, Structure, and Properties." The result is a collection of ten outstanding articles. These papers covered physicochemical properties of dietary fiber, its food applications, and novel fiber exploration. We believe these studies could make great contribution to dietary fiber in areas of both research and industrial applications.

Zhang et al. studied a novel dietary fiber material: bamboo shoot dietary fiber (BSDF). The effects of BSDF on the mechanical properties, moisture distribution, and microstructure of frozen dough were investigated. The results showed that the BSDF significantly improved the viscoelasticity and extensibility of frozen dough after thawing in a dose-dependent manner.
Wheat bran is rich in dietary fiber, which improves the flour nutritional content and also endows the flour with a richer flavor. However, a high content of insoluble dietary bran fiber may badly compromise the processing and edible quality of flour products. One study was conducted to explore ways to decrease the negative effects by using fermented bran, which showed longer dough extensibility and stability and better overall steam bread texture. This provided an innovative way to increase the dietary fiber content of steam bread.

$\mathrm{Li}$ et al. demonstrated how the complex additives including sodium carboxymethyl cellulose (CMC-Na) affect the storage property of steamed bread during frozen dough storage. The results showed that adding complex additives could enhance gas-holding capacity of gluten and maintain yeast activity upon frozen storage.

Another research was performed to investigate the quality of fresh wet noodles made from different flour milling streams. The basic composition, texture properties, cooking characteristics, and moisture status of the noodles were compared. The results indicated that as storage time increased, the springiness of fresh wet noodles gradually decreased, while the hardness increased.

Yang et al. reviewed the modification and application of dietary fiber in foods with respect to definition, classification, and methods for measurement, extraction, and modification. The supplementation of dietary fiber for flour, meat, and dairy products is also included. The benefits and risks of increasing consumption of dietary fiber are discussed. 


\section{Conflicts of Interest}

The editors declare that they have no conflicts of interest.

Qingbin Guo Ji Kang

Yanjie Bai

Feng Xu 

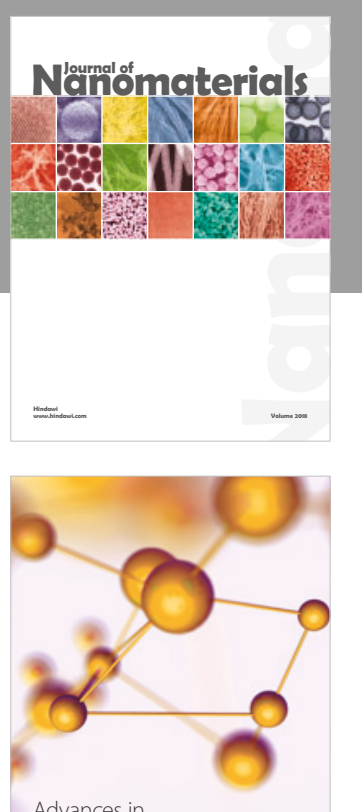

Physical Chemistry
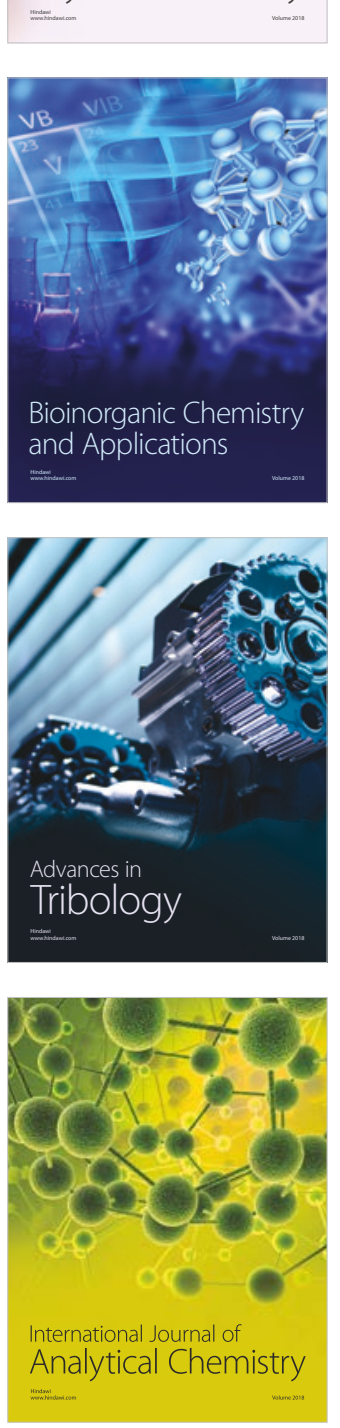

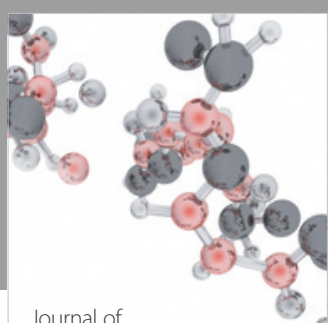

Analytical Methods

in Chemistry

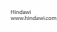

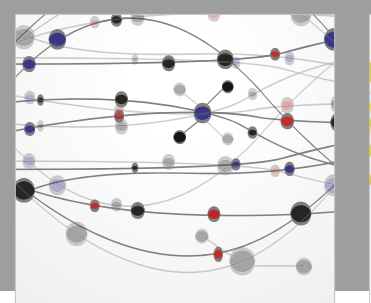

The Scientific World Journal

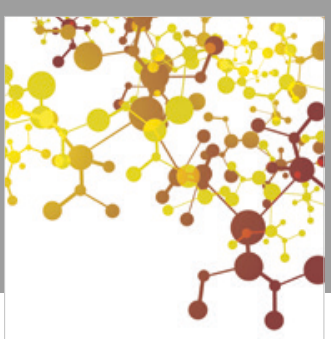

Journal of

Applied Chemistry
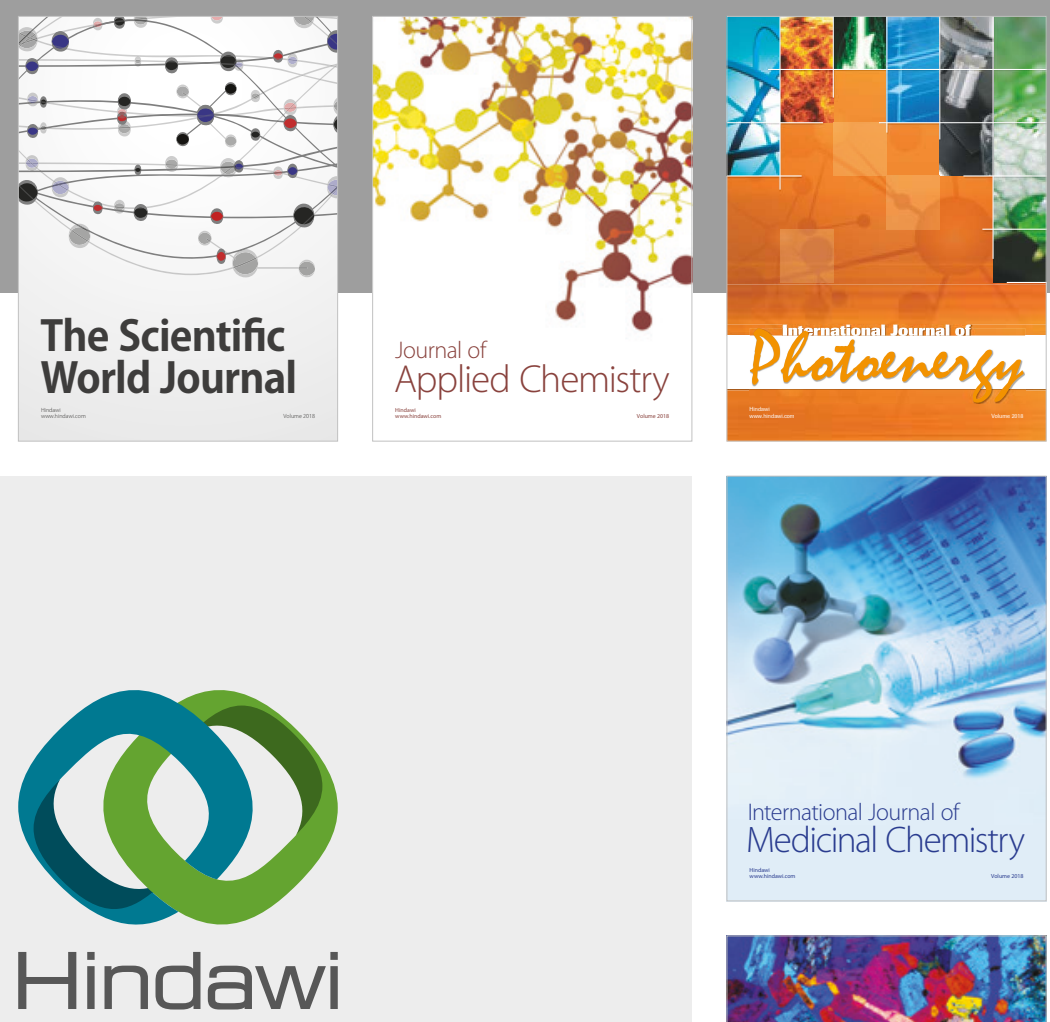

Submit your manuscripts at

www.hindawi.com
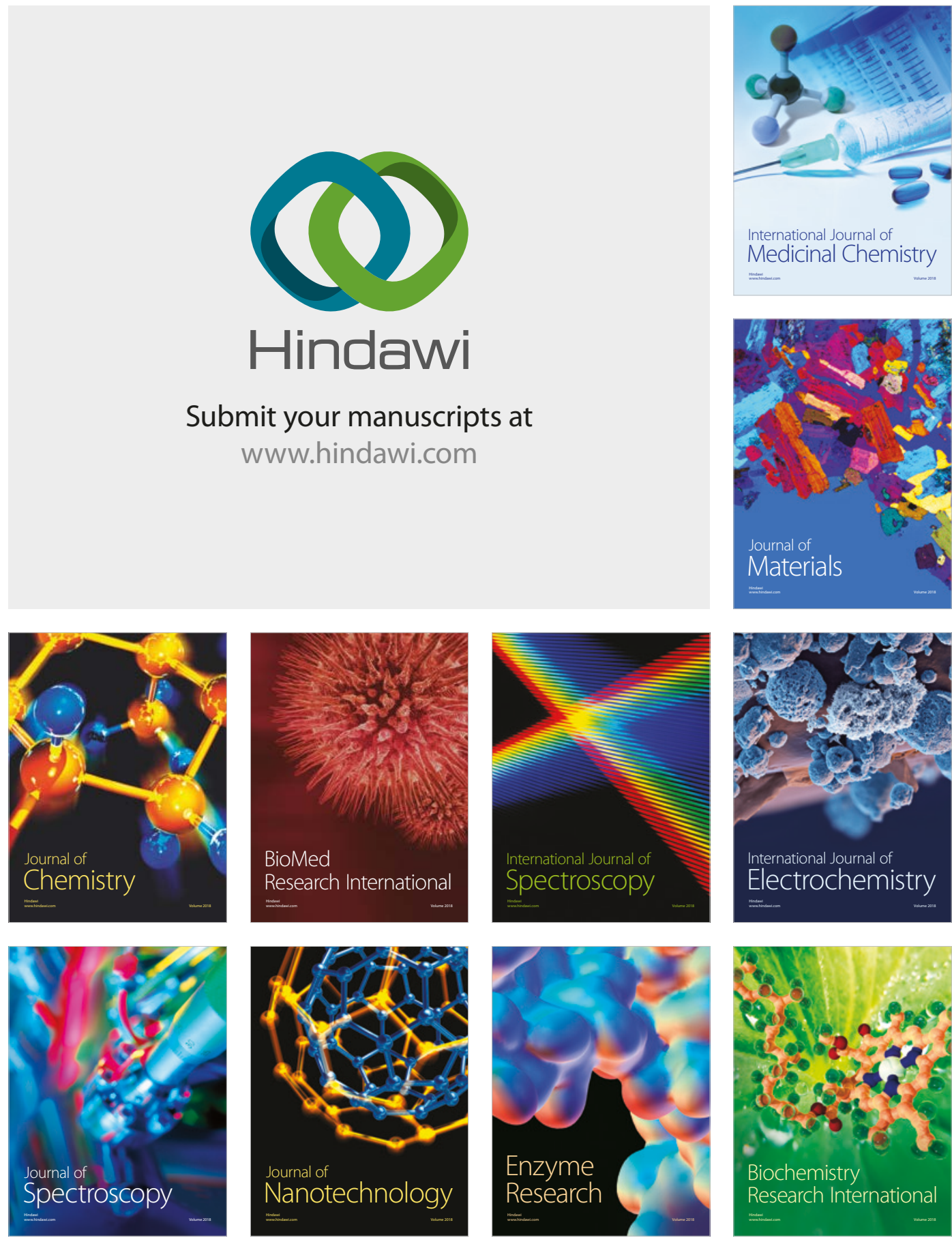
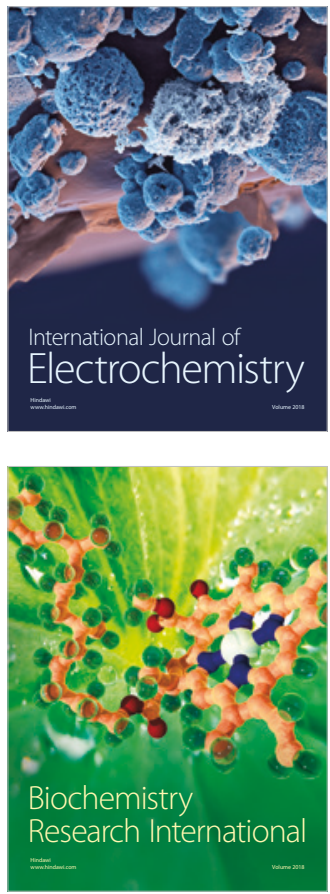